\title{
Approximate Equilibrium Problems and Fixed Points
}

\author{
H. Mazaheri ${ }^{1}$ and S. A. M. Mohsenalhosseini ${ }^{2}$ \\ ${ }^{1}$ Faculty of Mathematics, Yazd University, Yazd, Iran \\ ${ }^{2}$ Faculty of Mathematics, Vali-e-Asr University, Rafsanjan, Iran \\ Correspondence should be addressed to H. Mazaheri, hmazaheri@yazduni.ac.ir
}

Received 16 September 2012; Revised 21 November 2012; Accepted 12 December 2012

Academic Editor: Malte Braack

Copyright (C) 2013 H. Mazaheri and S. A. M. Mohsenalhosseini. This is an open access article distributed under the Creative Commons Attribution License, which permits unrestricted use, distribution, and reproduction in any medium, provided the original work is properly cited.

We find a common element of the set of fixed points of a map and the set of solutions of an approximate equilibrium problem in a Hilbert space. Then, we show that one of the sequences weakly converges. Also we obtain some theorems about equilibrium problems and fixed points.

\section{Introduction}

Equilibrium is "everywhere:" in economics, physics, engineering, chemistry, biology, and so forth. From the mathematical modelling point of view equilibrium can be described in fixed point theorems, optimisation problems, variational inequalities, complementarity problems, and so forth. Equilibrium systems can be studied from several points of view: existence of solutions; existence of nontrivial solutions; number of solutions; properties of the solution set; and the numerical approximation of solutions. In the first a short description of what is a mathematical equilibrium system in general, we will present several particular classes of approximate equilibrium systems and the relations between then.

Throughout the paper, let $H$ be a real Hilbert space; and let $C$ be a nonempty, closed, bounded, and convex subset of $H$.

Condition 1. The following condition appears implicitly in [1].

We assume that the map $\varphi: C \times C \rightarrow \mathbf{R}$ satisfies the following conditions:

(i) $\varphi(x, x)=0$, for all $x \in C$;

(ii) $\varphi$ is monotone, that is, $\varphi(x, y)+\varphi(y, x) \leq 0$, for all $x, y \in C$; (iii) for all $x, y \in C$,

$$
\lim _{t \rightarrow 0} \varphi(x t+(1-t) x, y) \leq \varphi(x, y) ;
$$

(iv) for each fixed $x \in C$, the function $y \mapsto \varphi(x, y)$ is convex and lower semicontinuous.

Condition 2. Assume that the map $\varphi: C \times C \rightarrow \mathbf{R}$ for $\epsilon>0$ satisfies the following conditions.

$\left(A_{1}\right) \varphi(x, x)=\epsilon$, for all $x \in C$.

$\left(A_{2}\right) \varphi$ is approximate monotone, that is, $\varphi(x, y)+\varphi(y, x) \leq$ $\epsilon$, for all $x, y \in C$.

$\left(A_{3}\right)$ For all $x, y \in C$,

$$
\lim _{t \rightarrow 0} \varphi(x t+(1-t) x, y) \leq \varphi(x, y) .
$$

$\left(A_{4}\right)$ For each fixed $x \in C$, the function $y \mapsto \varphi(x, y)$ is convex and lower semicontinuous.

Definition 1 (see [1]). We say that $x^{*} \in C$ is an equilibrium point of $\varphi: C \times C \rightarrow \mathbf{R}$ if there exists a $x^{*} \in C$, such that

$$
\varphi\left(x^{*}, y\right) \geq 0 \quad \forall y \in C ;
$$

the set of such $x^{*} \in C$ is denoted by $\operatorname{EP}(\varphi)$; that is,

$$
\operatorname{EP}(\varphi)=\left\{x^{*} \in C: \varphi\left(x^{*}, y\right) \geq 0, \forall y \in C\right\} .
$$


Definition 2. Suppose $\epsilon>0$, we say that $x^{*} \in C$ is an approximate equilibrium point of $\varphi: C \times C \rightarrow \mathbf{R}$ if there exists a $x^{*} \in C$, such that

$$
\varphi\left(x^{*}, y\right) \geq \epsilon \quad \forall y \in C .
$$

In this paper, the set of such an $x^{*} \in C$ is denoted by $\operatorname{AEP}(\varphi)$, that is,

$$
\operatorname{AEP}(\varphi)=\left\{x^{*} \in C: \varphi\left(x^{*}, y\right) \geq \epsilon, \forall y \in C\right\},
$$

and we set

$$
\begin{gathered}
\operatorname{AF}(T)=\{x \in C: d(x, T x)<\epsilon \text { for some } \epsilon>0\}, \\
F(T)=\{x \in C: T x=x\} .
\end{gathered}
$$

\section{Preliminaries}

In the following we will present a known lemma which is needed in the proof of some results (see [2]).

Lemma 3. Let $\varphi: C \times C \rightarrow \mathbf{R}$ be a map satisfies Condition 1. Let $r>0$, and let $x \in H$. Then there exists $a z \in C$, such that

$$
\varphi(z, y)+\frac{\langle y-z, z-x\rangle}{r} \geq 0 \quad \forall y \in C .
$$

Lemma 4. Let $\varphi: C \times C \rightarrow \mathbf{R}$ be a map satisfies Condition 2. Let $r>0, \epsilon>0$, and let $x \in H$. Then there exists $a z \in C$, such that

$$
\varphi(z, y)+\frac{\langle y-z, z-x\rangle}{r} \geq \epsilon \quad \forall y \in C .
$$

Proof. Consider the map $\psi: C \times C \rightarrow \mathbf{R}$ by $\psi(x, y)=$ $\varphi(x, y)-\epsilon$. The map $\psi$ satisfies Condition 1 . Then by Lemma 3 , there exists a $z \in C$, such that

$$
\psi(z, y)+\frac{\langle y-z, z-x\rangle}{r} \geq 0 \quad \forall y \in C .
$$

Thus

$$
\varphi(z, y)+\frac{\langle y-z, z-x\rangle}{r} \geq \epsilon \quad \forall y \in C .
$$

Lemma 5. Let $\varphi: C \times C \rightarrow \mathbf{R}$ be a map satisfies Condition 2. For $r>0, x \in H, \epsilon>0$ we defined $T_{r}: H \rightarrow C$, such that

$$
T_{r}(x)=\left\{z \in C \mid \varphi(z, y)+\frac{\langle y-z, z-x\rangle}{r} \geq \epsilon\right\} .
$$

\section{Then}

(a) $T_{r}$ is single valued.

(b) $T_{r}$ is firmly nonexpansive; that is,

$\left\|T_{r}(x)-T_{r}(y)\right\|^{2} \leq\left\langle T_{r}(x)-T_{r}(y), x-y\right\rangle \quad \forall x, y \in H$. (c) $F\left(T_{r}\right)=A E P(\varphi)$.

(d) AEP $(\varphi)$ is nonempty, closed, and convex.

Proof. (a) For $x \in H$ and $r>0$, let $z_{1}, z_{2} \in T_{r}(x)$. Then,

$$
\begin{gathered}
\varphi\left(z_{1}, y\right)+\frac{\left\langle y-z_{1}, z_{1}-x\right\rangle}{r} \geq \epsilon \quad \forall y \in C, \\
\varphi\left(z_{2}, y\right)+\frac{\left\langle y-z_{2}, z_{2}-x\right\rangle}{r} \geq \epsilon \quad \forall y \in C .
\end{gathered}
$$

Then

$$
\begin{aligned}
& \varphi\left(z_{1}, z_{2}\right) \geq \frac{\left\langle z_{2}-z_{1}, x-z_{1}\right\rangle}{r}+\epsilon, \\
& \varphi\left(z_{2}, z_{1}\right) \geq \frac{\left\langle z_{1}-z_{2}, x-z_{2}\right\rangle}{r}+\epsilon .
\end{aligned}
$$

Since $\varphi$ is approximate monotone,

$$
\begin{aligned}
\epsilon & \geq \varphi\left(z_{1}, z_{2}\right)+\varphi\left(z_{2}, z_{1}\right) \\
& \geq \frac{\left\langle z_{2}-z_{1}, x-z_{1}\right\rangle}{r}+\frac{\left\langle z_{1}-z_{2}, x-z_{2}\right\rangle}{r}+2 \epsilon \\
& \geq \frac{\left\langle z_{2}-z_{1}, z_{1}-z_{2}\right\rangle}{r}+\epsilon .
\end{aligned}
$$

Now, since $\epsilon>0$ and $r>0$, we have

$$
\left\langle z_{2}-z_{1}, z_{1}-z_{2}\right\rangle \leq 0
$$

So, we have $z_{1}=z_{2}$.

(b) Now we claim that $T_{r}$ is a firmly nonexpansive. Indeed, if $x, y \in H$,

$$
\begin{gathered}
\varphi\left(T_{r}(x), T_{r}(y)\right)+\frac{\left\langle T_{r}(y)-T_{r}(x), T_{r}(x)-x\right\rangle}{r} \geq \epsilon, \\
\varphi\left(T_{r}(y), T_{r}(x)\right)+\frac{\left\langle T_{r}(x)-T_{r}(y), T_{r}(y)-y\right\rangle}{r} \geq \epsilon .
\end{gathered}
$$

Adding the two inequalities, we have

$$
\begin{aligned}
& \varphi\left(T_{r}(x), T_{r}(y)\right)+\varphi\left(T_{r}(y), T_{r}(x)\right) \\
& \quad+\frac{\left\langle T_{r}(y)-T_{r}(x), T_{r}(x)-T_{r}(y)-x+y\right\rangle}{r} \\
& \quad \geq 2 \epsilon .
\end{aligned}
$$

With $\left(A_{2}\right)$, we have

$$
\frac{\left\langle T_{r}(y)-T_{r}(x), T_{r}(x)-T_{r}(y)-(x-y)\right\rangle}{r} \geq \epsilon .
$$

Now since $\epsilon>0$ and $r>0$, then

$$
\left\langle T_{r}(y)-T_{r}(x), T_{r}(x)-T_{r}(y)-(x-y)\right\rangle \geq 0
$$

so

$$
\left\|T_{r}(x)-T_{r}(y)\right\|^{2} \leq\left\langle T_{r}(x)-T_{r}(y), x-y\right\rangle .
$$


(c) Take $x \in K$. Then

$$
\begin{aligned}
x \in F\left(T_{r}\right) & \longrightarrow x=T_{r}(x) \\
& \longrightarrow \varphi(x, y)+\frac{\langle x-x, y-x\rangle}{r} \geq \epsilon \quad \forall y \in C \\
& \longrightarrow \varphi(x, y) \geq \epsilon \quad \forall y \in C \\
& \longrightarrow x \in \operatorname{AEP}(\varphi) .
\end{aligned}
$$

(d) At last, we claim that $\operatorname{AEP}(\varphi)$ is closed and convex. Indeed, since $T_{r}$ is firmly nonexpansive, $T_{r}$ is also nonexpansive, and since the fixed-point set of a nonexpansive operator is closed and convex [3, proposition 1.5.3]. Therefore follows from (b), (c).

In the following we will present a known theorem which is needed in the proof of some results (see [4]).

Theorem 6. Let $\varphi: C \times C \rightarrow \mathbf{R}$ be a map satisfies Condition 1 , and let $S: C \rightarrow C$ be a nonexpentive mapping such that $F(S) \cap \operatorname{EP}(\varphi) \neq \varnothing$. Let $\left\{x_{n}\right\}$ and $\left\{u_{n}\right\}$ be sequences generated initially by an arbitrary element $x_{1} \in H$ and then by

$$
\begin{gathered}
\varphi\left(u_{n}, y\right)+\frac{\left\langle y-u_{n}, u_{n}-x_{n}\right\rangle}{r_{n}} \geq 0 \quad \forall y \in C, \\
x_{n+1}=\alpha_{n} u_{n}+\left(1-\alpha_{n}\right) S u_{n} \quad \forall n \geq 1,
\end{gathered}
$$

where $\left\{\alpha_{n}\right\}$ and $\left\{r_{n}\right\}$ satisfy the following conditions:

(i) $\left\{\alpha_{n}\right\} \subset[\alpha, \beta]$ for some $\alpha, \beta \in(0,1)$;

(ii) $\left\{r_{n}\right\} \subset(0, \infty)$ and $\lim \inf _{n} r_{n}>0$.

Then, the sequences $\left\{x_{n}\right\}$ and $\left\{u_{n}\right\}$ converge weakly to an element of $F(S) \cap E P(\varphi) \neq \varnothing$.

Lemma 7. Let $\psi: C \times C \rightarrow \mathbf{R}$ be a map by $\psi(x, y)=$ $\varphi(x, y)-\epsilon$ that satisfies Condition 1 , and let $S: C \rightarrow C$ be a nonexpentive mapping, then $F(S) \cap A E P(\varphi) \neq \varnothing$ if and only if $F(S) \cap E P(\psi) \neq \varnothing$.

In the following we will present a theorem which is extended Theorem 6 .

Theorem 8. Let $\varphi: C \times C \rightarrow \mathbf{R}$ be a map satisfies Condition 2, and let $S: C \rightarrow C$ be a map such that $F(S) \cap A E P(\varphi) \neq \varnothing$. Let $\left\{x_{n}\right\}$ and $\left\{u_{n}\right\}$ be sequences generated initially by an arbitrary element $x_{1} \in H$ and then by

$$
\begin{gathered}
\varphi\left(u_{n}, y\right)+\frac{\left\langle y-u_{n}, u_{n}-x_{n}\right\rangle}{r_{n}} \geq \epsilon \quad \forall y \in C, \\
x_{n+1}=\alpha_{n} u_{n}+\left(1-\alpha_{n}\right) S u_{n} \quad \forall n \geq 1,
\end{gathered}
$$

where $\left\{\alpha_{n}\right\}$ and $\left\{r_{n}\right\}$ satisfy the following conditions:

(i) $\left\{\alpha_{n}\right\} \subset[\alpha, \beta]$ for some $\alpha, \beta \in(0,1)$;

(ii) $\left\{r_{n}\right\} \subset(0, \infty)$ and $\lim \inf _{n} r_{n}>0$.
Then, the sequences $\left\{x_{n}\right\}$ and $\left\{u_{n}\right\}$ converge weakly to an element of $F(S) \cap A E P(\varphi)$.

Proof. Suppose the nap $\psi: C \times C \rightarrow \mathbf{R}$ by $\psi(x, y)=$ $\varphi(x, y)-\epsilon$ that satisfies Condition 1 . Since by $F(S) \cap \operatorname{AEP}(\varphi)$ $\neq \varnothing$, Lemma 9 implies that $F(S) \cap \operatorname{EP}(\psi) \neq \varnothing$ and holds conditions theorem (2.8), therefore the sequences $\left\{x_{n}\right\}$ and $\left\{u_{n}\right\}$ converge weakly to an element of $z \in F(S) \cap \operatorname{EP}(\psi)$. By Lemma 9 the sequences $\left\{x_{n}\right\}$ and $\left\{u_{n}\right\}$ converge weakly to $z \in F(S) \cap \operatorname{AEP}(\varphi)$.

Lemma 9. Let $\varphi: C \times C \rightarrow \mathbf{R}$ be a map satisfies Condition 1. For $x \in C$, define a mapping $T: C \rightarrow C$ such that is contraction. If $\varphi(T x, y) \geq 0$ for all $y \in C$, then there exists $x_{0} \in E P(T)$.

Proof. Since $C$ is a nonempty closed, bounded, and convex subset of $H$, and $T$ is continues, then by Browde's theorem there exists a $x_{0} \in C: T x_{0}=x_{0}$. Since $\varphi\left(T x_{0}, y\right) \geq 0$ for all $y \in C$, thus $\varphi\left(x_{0}, y\right) \geq 0$ for all $y \in C$. So $\exists x_{0} \in C: x_{0} \in$ $\mathrm{EP}(T)$.

Lemma 10. Let $\varphi: C \times C \rightarrow \mathbf{R}$ be a map satisfies Condition 1. For $x \in C$, define a mapping $T: C \rightarrow C$ such that is contraction. If $\varphi\left(T^{n} x, y\right) \geq 0$, for all $y \in C$, then $x_{0} \in \operatorname{EP}(T)$.

Proof. Since $C$ is a nonempty closed, bounded, and convex subset of $H$, and $T$ is contraction, now by Theorem 2.1 of [4], since $\left\{T^{n} x\right\}$ converges to fixed point $T$, then there exists a $x_{0} \in$ $C: T^{n} x_{0}=x_{0}$. Since for all $y \in C, \varphi\left(T^{n} x_{0}, y\right) \geq 0$, thus $\varphi\left(x_{0}, y\right) \geq 0$ for all $y \in C$. So there exists a $x_{0} \in C$, such that $x_{0} \in \operatorname{EP}(T)$.

Lemma 11. Let $\varphi: C \rightarrow \mathbf{R}$ be a real-valued function, let $\varphi: C \times C \rightarrow \mathbf{R}$ be a map satisfies Condition 2, and let $T:$ $C \rightarrow C$ be a nonlinear onto mapping and satisfying

$$
\|x-T x\|+\varphi(x, T x)<\epsilon \quad \forall x \in C .
$$

If $\operatorname{EP}(\varphi) \neq \varnothing$, then $A F(T) \neq \varnothing$.

Proof. If $z \in \mathrm{EP}(\varphi)$, then

$$
0 \leq \varphi(z, y) \quad \forall y \in C .
$$

Since $z \in C$, there exists $x \in C$, such that $T x=z$. Therefore $\varphi(T x, x) \geq 0$, and so

$$
d(x, T x)+\varphi(x, T x)<\epsilon .
$$

It follows that $d(x, T x)<\epsilon$ and $x \in \operatorname{AF}(T)$.

Lemma 12. Let the map $\varphi: C \times C \rightarrow \mathbf{R}$ satisfy Condition 2 . For $x \in C$, define a mapping $T: C \rightarrow C$ such that is contraction. If $\varphi(x, T x)<d(x, T x)$, for all $x \in C$, then there exists a $x_{0} \in A E P(T)$.

Proof. Since $T$ is contraction by Theorem 2.1. of [1] AF $(T) \neq$ $\varnothing$, for all $\epsilon>0$, and since for all $x \in C \varphi(x, T x)<\|x-T x\|$, therefore for all $x \in C$ and for some $\epsilon>0, \varphi(x, T x)<\epsilon$, for all $x \in C$. Thus $x_{0} \in \operatorname{AEP}(T)$. 


\section{References}

[1] L.-C. Ceng, S. Al-Homidan, Q. H. Ansari, and J.-C. Yao, "An iterative scheme for equilibrium problems and fixed point problems of strict pseudo-contraction mappings," Journal of Computational and Applied Mathematics, vol. 223, no. 2, pp. 967-974, 2009.

[2] E. Blum and W. Oettli, "From optimization and variational inequalities to equilibrium problems," The Mathematics Student, vol. 63, no. 1-4, pp. 123-145, 1994.

[3] K. Goebel and S. Reich, Uniform Convexity, Hyperbolic Geometry, and Nonexpansive Mappings, vol. 83 of Monographs and Textbooks in Pure and Applied Mathematics, Marcel Dekker Inc., New York, NY, USA, 1984.

[4] L. C. Ceng, N. C. Wong, and J. C. Yao, "Implicit predictorcorrector iteration process for finitely many asymptotically (quasi-)nonexpansive mappings," Journal of Inequalities and Applications, vol. 2006, Article ID 65983, 11 pages, 2006. 


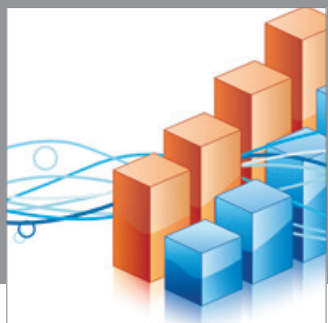

Advances in

Operations Research

mansans

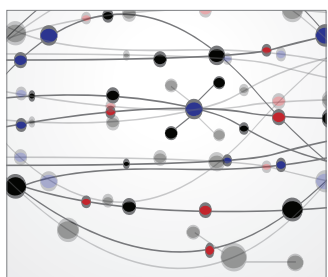

The Scientific World Journal
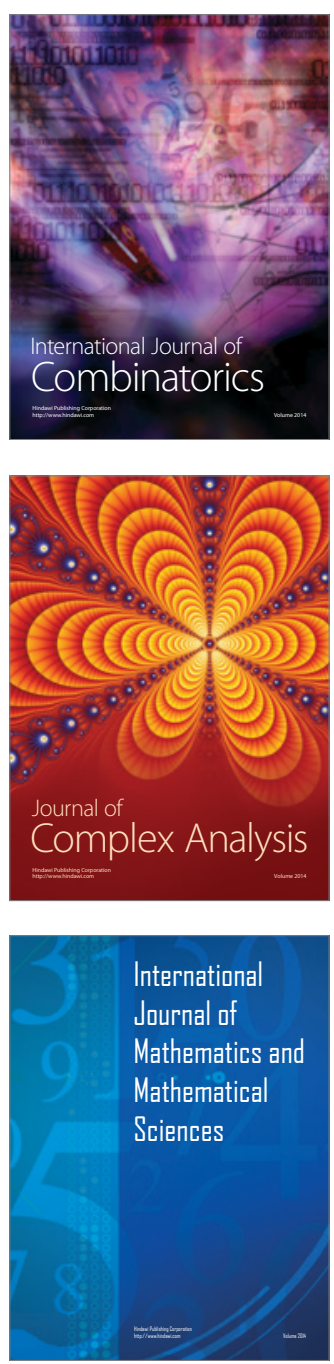
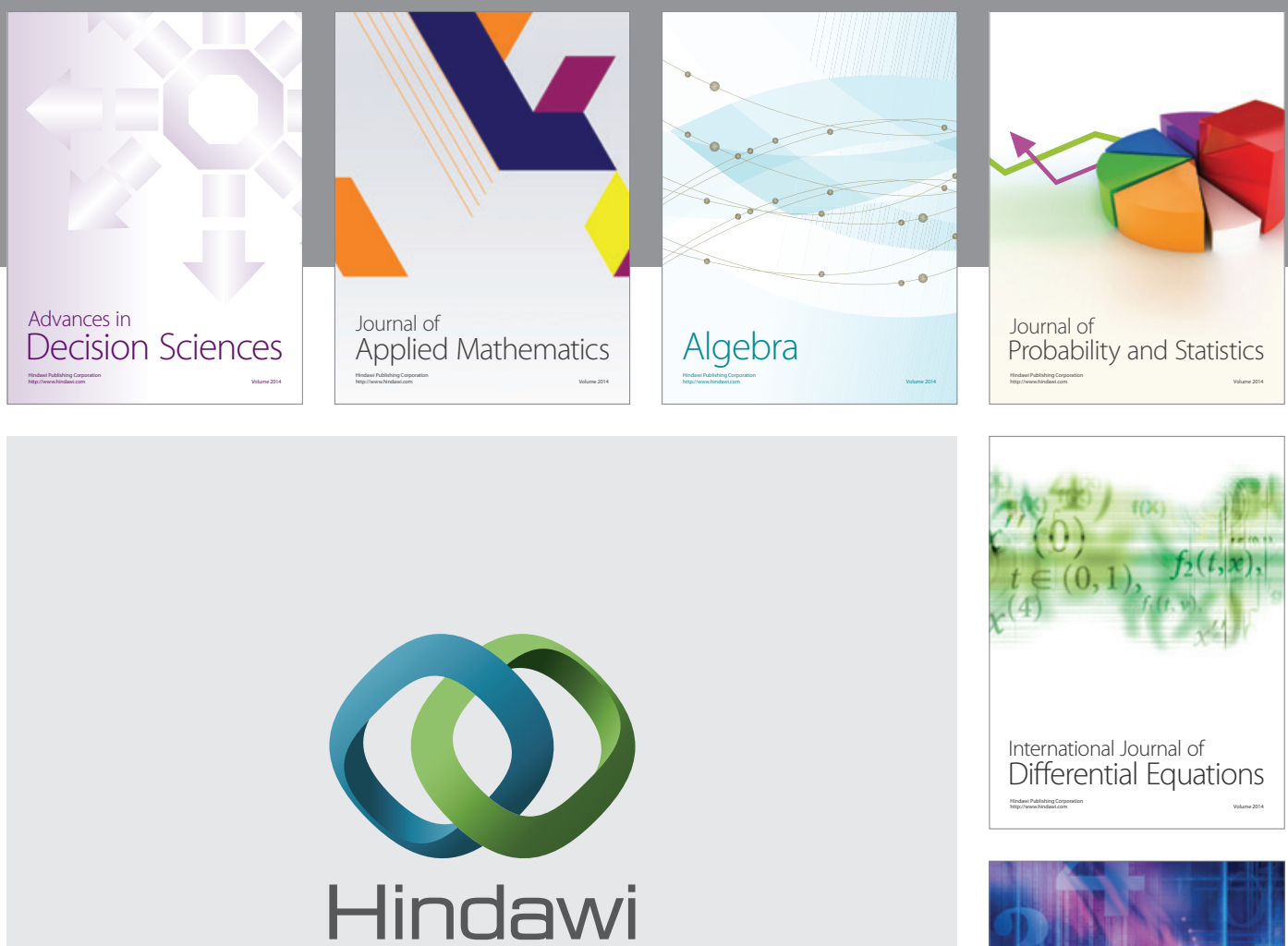

Submit your manuscripts at http://www.hindawi.com
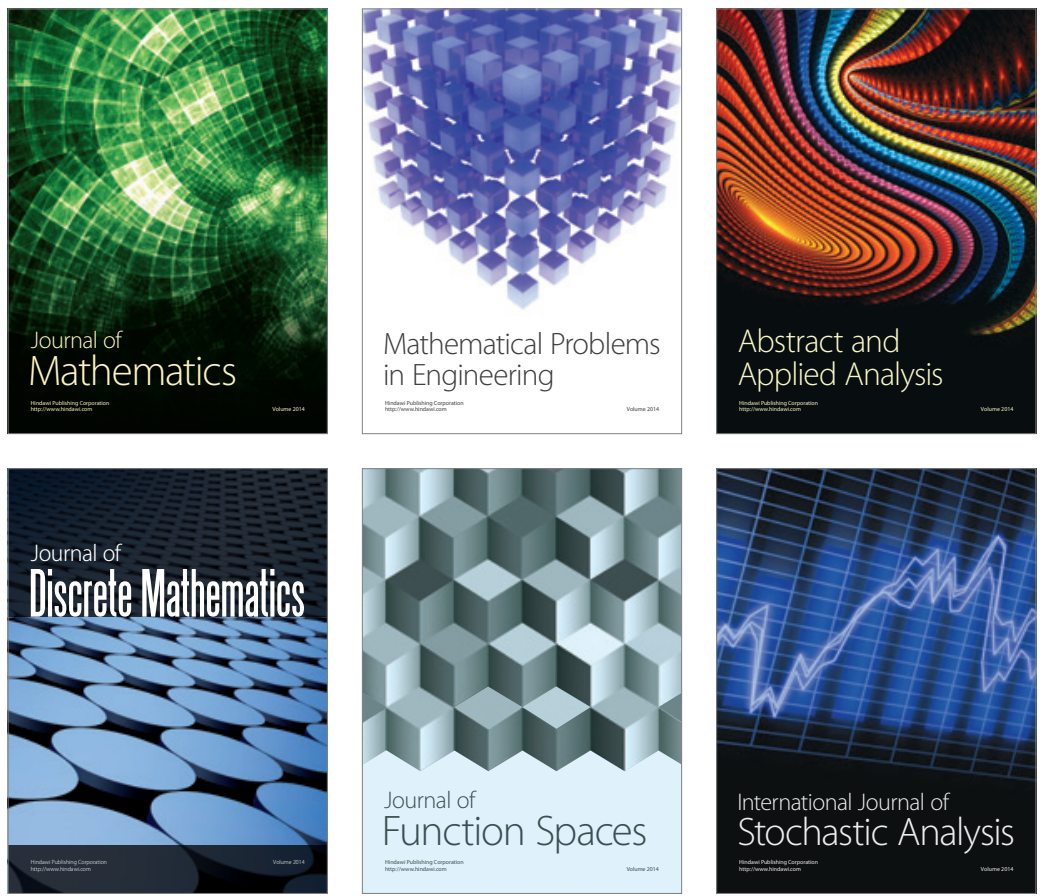

Journal of

Function Spaces

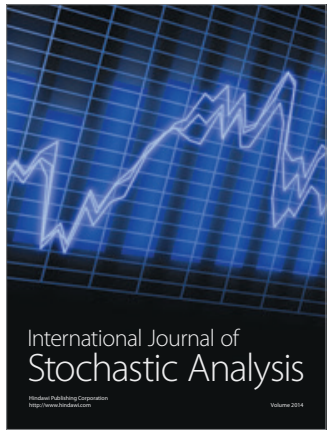

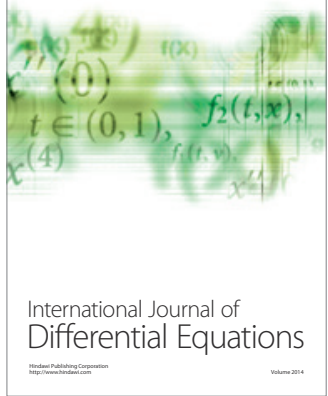
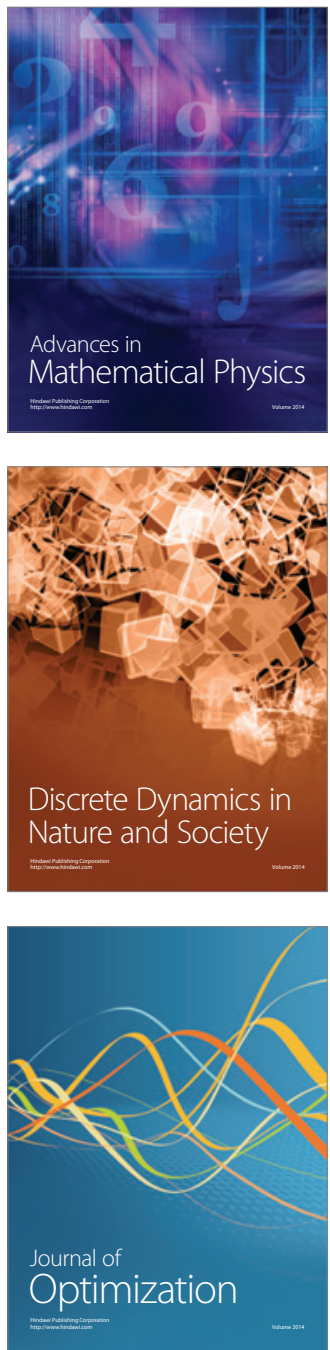